\title{
Type 2 Diabetes, Metabolic Syndrome and Lipid Metabolism
}

\author{
Orit Pinhas-Hamiel \\ Pediatric Endocrinology and Diabetes Unit, Edmond and Lily Safra Children's Hospital, Sheba Medical Center, \\ Ramat-Gan, and Juvenile Diabetes Center, Maccabi Health Care Services, Tel-Aviv University Sackler School of \\ Medicine, Tel-Aviv, Israel
}

\begin{abstract}
This year, a 5-year-old girl became one of the youngest individuals diagnosed with type 2 diabetes (T2DM). A study from the USA revealed an overall prevalence of T2DM of $0.36 \%$, including an estimated 120,000 adolescents, about one third of whom are undiagnosed. These numbers are alarming considering findings on the safety and tolerability of current treatments for youth with T2DM and the recent finding of high rates of morbidity and mortality. Interestingly, glycemic control alone was found to be insufficient to attenuate the excess risk in T2DM. Evidence suggests that features of the metabolic syndrome (MetS) may be involved in the pathogenesis of co-morbidities.

Genetic mutations causing an inherited form of the MetS were identified, with implications for drug development across diabetes, heart disease, and obesity. Metformin was found to increase lifespan by altering microbial folate and methionine metabolism.

The 2013 guidelines of the American College of Cardiology and American Heart Association (ACCAHA) for the management of cholesterol, released in November, are reviewed, as well as a comment on the controversies that emerged after their publication regarding their implications for public health. The results of 1-year treatment with a PCSK9 inhibitor are reviewed, as well as the results of a trial of mipomersen, a new second-generation apolipoprotein B antisense oligonucleotide. Finally, the effect of 'an apple a day' versus statin therapy is compared.
\end{abstract}

\section{Type 2 diabetes mellitus}

New concerns

\section{A 5-year-old girl with type 2 diabetes}

Kevat D, Wilson D, Sinha A

Cairns Hospital and Diabetes Centre, Cairns, QLD, and School of Public Health, Monash University, Melbourne, VIC, Australia

dev.kevat@monash.edu

Lancet 2014;383:1268

A 5 -year-old obese indigenous girl presented with non-healing sores on her thighs. Random blood glucose level tests showed concentrations of 19.2 and $18.7 \mathrm{mmol} / \mathrm{l}$. A urine dipstick test was negative for ketones. There was a history of bedwetting for the past 12 months. The child was born macrosomic $(4.5 \mathrm{~kg})$ at 38 weeks by caesarean section after a pregnancy complicated by poorly controlled maternal diabetes. There was a strong family history of T2DM. The patient was above the 95th centile for weight $(36 \mathrm{~kg})$ and body mass index (24.5). She had marked acanthosis nigricans in the axillae and around the neck. Her diet was high in refined carbohydrates and simple sugars. $\mathrm{HbA}_{1 \mathrm{c}}$ level was $11.9 \%$, C-peptide $1.6 \mathrm{mmol} / \mathrm{l}$ (normal range 0.3-1.4), and insulin $201 \mathrm{pmol} / \mathrm{l}$ (14-160). Tests for T1DM autoantibodies and genetic tests for MODY1 (HNF4A) and MODY3 (HNF1A) were negative.

A 5-year-old indigenous girl from a remote community in far north Queensland was diagnosed with T2DM. Her weight is $36 \mathrm{~kg}$, equivalent to the 50th percentile weight of an 11-year-old girl. Her BMI is 24 , which is 2.8 SDs above the average for her age and gender. She has all the risk factors associated with T2DM: a positive family history, exposure to maternal diabetes in utero (which is associated with a 5.7-fold risk for T2DM in adolescence [1]), high birth weight and poor diet. She is morbidly obese and has signs of hyperinsulinism such as acanthosis nigricans. The prevalence of T2DM is known to be much greater among indigenous children and adolescents than among other young people. However, 
the onset of T2DM typically occurs during early adolescence. The young age of this girl is extremely unusual. The youngest age at T2DM diagnosis among Pima Indians was 3.5 years [2]. We can only hope that one swallow does not make a summer. Increased lifetime exposure to hyperglycemia predicts a high complication risk. This case underscores the need for very early intervention to prevent T2DM among children.

\section{Important for clinical practice}

\section{Prevalence of diagnosed and undiagnosed type 2 diabetes mellitus among US adolescents: results from the continuous NHANES, 1999-2010}

Demmer RT, Zuk AM, Rosenbaum M, Desvarieux M

Am J Epidemiol 2013;178:1106-1113

Introduction: The prevalence and incidence of T2DM are reportedly increasing among adolescents, however national data are lacking, particularly with regard to undiagnosed T2DM. This study aimed to estimate the prevalence of diagnosed and undiagnosed T2DM among US adolescents.

Methods: A nationally representative cross section of 11,888 adolescents aged 12-19 years who received a diabetes interview in the Continuous National Health and Nutrition Examination Survey during 1999-2010 was analyzed. In addition, a random subsample of 4,661 adolescents had fasting blood samples collected. Persons who reported a previous diabetes diagnosis and were either taking no medication or taking an oral hypoglycemic agent (with or without insulin) were classified as having T2DM; persons who reported using insulin alone were classified as having type 1 diabetes (T1DM). Undiagnosed diabetes was defined as a fasting plasma glucose concentration of $\geq 126 \mathrm{mg} / \mathrm{dl}$ and was assumed to be T2DM.

Results: In the fasting subsample, 31 known diabetes cases (T1DM and T2DM) were identified, representing a prevalence of $0.84 \%$. Estimates of the prevalences of T1DM and T2DM were 0.48 and $0.36 \%$, respectively, indicating that T2DM accounted for $43 \%$ of all cases. Further, undiagnosed T2DM prevalence was $0.12 \%$, representing $34 \%$ of T2DM cases.

Conclusions: T2DM accounts for approximately half of adolescent diabetes in the United States, and one third of these cases are undiagnosed.

Although evidence suggests that the prevalence of T2DM has been increasing among US adolescents as a result of the obesity epidemic, there are very few reliable estimates from representative US adolescent populations. The current study analyzed data from six nationally representative serial cross sections of US adolescents enrolled in the continuous National Health and Nutrition Examination Survey (NHANES) during a 12-year period from 1999 to 2010, comprising 32,676,471 US adolescents. The study reports both the prevalence of T2DM based on questionnaires and the prevalence of undiagnosed diabetes based on fasting glucose measurements. The classification of diabetes in this study differs from previously used epidemiologic definitions of diabetes in that participants receiving both insulin and oral hypoglycemic agents were classified as having T2DM, whereas in previous studies all subjects receiving any insulin therapy had been classified as having T1DM.

The prevalence of diagnosed T2DM was 1.8 cases per 1,000 adolescents and for undiagnosed T2DM was 1.2 cases per 1,000 adolescents. The overall prevalence of T2DM of $0.36 \%$ represents an estimated 119,224 cases of adolescents with T2DM in the USA, about one third ( $>40,000$ cases) of whom are undiagnosed. The study may have limitations regarding diabetes case definition and etiology classification. Indeed, in the SEARCH study (including only youth with diagnosed diabetes), the prevalence of T2DM among adolescents aged 10 through 19 years was much lower, though the increase during an 8-year period was dramatic, from 0.34 per 1,000 in 2001 to 0.46 per 1,000 in 2009 [3]. 


\section{Safety and tolerability of the treatment of youth-onset type 2 diabetes: the TODAY experience}

TODAY Study Group

Diabetes Care 2013;36:1765-1771

Objective: Data related to the safety and tolerability of treatments for pediatric T2DM are limited. The TODAY clinical trial assessed severe adverse events (SAEs) and targeted non-severe adverse events (AEs) before and after treatment failure, which was the primary outcome (PO).

Methods: Obese 10 - to 17 -year-olds $(\mathrm{n}=699)$ with $\mathrm{T} 2 \mathrm{DM}$ for $<2$ years and $\mathrm{HbA}_{1 \mathrm{c}} \leq 8 \%$ on metformin monotherapy were randomized to one of three treatments: metformin alone, metformin + rosiglitazone $(\mathrm{M}+\mathrm{R})$, or metformin + lifestyle program $(\mathrm{M}+\mathrm{L})$. Participants were followed for 2-6.5 years.

Results: Gastrointestinal disturbance was the most common AE (41\%) and was lowest in the M+R group $(\mathrm{p}=0.018)$. Other common AEs included anemia ( $20 \%$ before primary outcome), abnormal liver transaminases $(16 \%)$, excessive weight gain $(7 \%)$, and psychological events $(10 \%)$; the AEs were similar across treatments. Permanent medication reductions/discontinuations occurred most often because of abnormal liver transaminases and were lowest in the $M+R$ group $(p=0.005)$. Treatment-emergent SAEs were uncommon and similar across treatments. Most $(98 \%)$ were unrelated or unlikely related to the study intervention. There were no deaths and only 18 targeted SAEs (diabetic ketoacidosis, $\mathrm{n}=12$; severe hypoglycemia, $\mathrm{n}=5$; lactic acidosis, $\mathrm{n}=1$ ). 62 pregnancies occurred in 45 participants, and 6 offspring had congenital anomalies.

Conclusions: The TODAY study represents extensive experience managing T2DM in youth and found that the three treatment approaches were generally safe and well tolerated. Adding rosiglitazone to metformin may reduce gastrointestinal side effects and hepatotoxicity.

Only metformin and insulin are currently approved by the Food and Drug Administration for treatment of T2DM in children. Long-term experience to define the efficacy and safety of treatment approaches is limited. The Treatment Options for type 2 Diabetes in Adolescents and Youth (TODAY) clinical trial was designed to address the safety and efficacy of three distinct approaches. Particular attention was paid to the side effects of metformin (gastrointestinal symptoms, anemia, lactic acidosis) and rosiglitazone (hepatotoxicity, anemia, edema, weight gain, heart failure, fractures) previously reported in adults. The ability to complete a 2- to 6-month run-in period, taking metformin monotherapy and $\mathrm{HbA}_{1 \mathrm{c}}<8 \%$ were inclusion criteria. Participants, aged 10-17 years, were randomized (1:1:1) to one of three groups: (1) metformin alone (1,000 mg twice daily); (2) metformin + rosiglitazone ( $4 \mathrm{mg}$ twice daily), and (3) metformin + intensive lifestyle intervention. The primary outcome was time to treatment failure, defined as either $\mathrm{HbA}_{1 \mathrm{c}} \geq 8 \%$ sustained for 6 months or the inability to wean from insulin within 3 months after acute metabolic decompensation [4].

A number of important issues arise from this study. The authors concluded that the three treatment approaches are all generally safe and well tolerated. However, there were overall 688 reported adverse events ( $40 \%$ gastrointestinal, $7 \%$ excessive weight gain, $10 \%$ psychological events, $20 \%$ anemia). Serious adverse events accounted for 117 events (17\%), although most were considered unrelated to study participation. Due to the adverse effects, $10 \%$ discontinued or had dose reduction of the medications. Notably, $45 \%$ of the participants reached the primary outcome, treatment failure. Considering that participants had only short disease duration (mean 7.8 months) and that the mean $\mathrm{HbA}_{1 \mathrm{c}}$ was $7.1 \%$ at recruitment, the worsening $\mathrm{HbA}_{1 \mathrm{c}}$ under treatment is alarming. It is possible that participants did not adhere to the treatment or that the side effects negatively impacted their compliance. There are numerous barriers to medication adherence among chronically ill adolescents including forgetfulness, organizational demands, and financial costs [5]. In addition, a greater number of medications prescribed and adverse drug reactions are known causes for poor adherence. This study highlights the difficulties involved in treating adolescents with T2DM.

Rosiglitazone is no longer an option for young patients with T2DM because of concerns about the cardiovascular and other adverse effects of this class of medications. I encourage you to read the commentary 'Crisis in Care: Limited Treatment Options for Type 2 Diabetes in Adolescents and Youth' on the major obstacles to the approval of new drugs for the treatment of youth with T2DM [6]. 


\section{Long-term complications and mortality in young-onset diabetes: type 2 diabetes is more hazardous and lethal than type 1 diabetes}

Constantino MI, Molyneaux L, Limacher-Gisler F, Al-Saeed A, Luo C, Wu T, Twigg SM, Yue DK, Wong J

maria.constantino@sswahs.nsw.gov.au

Diabetes Care 2013;36:3863-3869

Objective: To evaluate long-term clinical outcomes and survival in young-onset T2DM compared with T1DM with a similar age of onset.

Methods: Clinical and mortality outcomes in 354 patients with T2DM, age of onset between 15 and 30 years (T2DM15-30), were compared with 470 patients with T1DM with a similar age of onset (T1DM15-30).

Results: During a median observation period of 21.4 (IQR 14-30.7) and 23.4 (15.7-32.4) years for the T2DM and T1DM cohorts, respectively, 71 of 824 patients $(8.6 \%)$ died. A significant mortality excess was noted in T2DM15-30 (11 vs. $6.8 \%, \mathrm{p}=0.03)$, with an increased hazard for death (hazard ratio $2.0, \mathrm{p}=0.003)$. Death for T2DM15-30 occurred after a shorter disease duration $(26.9(18.1-36.0)$ vs. $36.5(24.4-45.4)$ years, $\mathrm{p}=0.01)$ and at a younger age. There were more cardiovascular deaths in T2DM15-30 (50 vs. 30\%, p < 0.05). Despite equivalent glycemic control and shorter disease duration, the prevalence of albuminuria and less favorable cardiovascular risk factors were greater in the T2DM15-30 cohort, even soon after diabetes onset. Neuropathy scores and macrovascular complications were also higher in T2DM15-30 ( $\mathrm{p}<0.0001)$.

Conclusions: Young-onset T2DM is the more lethal phenotype of diabetes and is associated with a greater mortality, more diabetes complications, and unfavorable cardiovascular disease risk factors when compared with T1DM.

Data on the long-term morbidity and survival of youth with T2DM are scarce. In the current study, clinical and mortality outcomes were established by matching records of youth with T2DM with those of T1DM. Mortality rates were derived from the Australian National Death Index for the period: 1986-2011. Over a median observation period of about 20 years, the mortality rate was 2 -fold higher in the T2DM group. Moreover, deaths in the T2DM group occurred after a shorter median disease duration, and at a younger age ( $53 \pm 15$ vs. $57 \pm 12$ years). Because ethnicity differed between the groups, outcomes were also compared among Anglo-Celtic subjects only: differences in mortality rates persisted in this sub-analysis.

Importantly, there was no difference in $\mathrm{HbA}_{1 \mathrm{c}}$ levels between the groups, suggesting that good glycemic control is not enough to attenuate the excess risk in T2DM. Albuminuria, CVD risk factors, peripheral neuropathy and macrovascular complications presented in the T2DM group as early as 2.5 years after diabetes onset. The constellation of higher BMI, dyslipidemia, hypertension, and elevated urine albumin/creatinine ratio, all seen in the 20-year age group, may contribute to the early onset of cardiovascular morbidity. The authors suggest that the diagnosis of T2DM, rather than T1DM, is often met with relief. Patients believe that because they do not need insulin injections, their disease is not as serious. However, these and other findings demonstrate the opposite. T2DM in youth is an aggressive disease, even more so than T1DM. It is possible that caregivers and physicians also treat T2DM as less serious than T1DM. While patients with T1DM receive intensive insulin treatment, insulin pumps, continuous glucose sensors and frequent clinical visits, those with T2DM may be less intensively treated, as reflected by low use of ACE inhibitors, angiotensin receptor blockers and statins. The editorial accompanying this paper called for a clinical trial evaluating CVD prevention in young people with T1DM and T2DM, focusing on intensive blood pressure and lipid control. 


\section{Metformin retards aging in Caenorhabditis elegans by altering microbial folate and methionine metabolism}

Cabreiro F, Au C, Leung KY, Vergara-Irigaray N, Cocheme HM, Noori T, Weinkove D, Schuster E, Greene ND, Gems D Institute of Healthy Ageing, and GEE, University College London, London, UK

Cell 2013;153:228-239

Background: The biguanide drug metformin is widely prescribed to treat T2DM and MetS, but its mode of action remains uncertain. Metformin also increases lifespan in Caenorhabditis elegans co-cultured with Escherichia coli. This bacterium exerts complex nutritional and pathogenic effects on its nematode predator/host that impact health and aging.

Results: Metformin increased lifespan by altering microbial folate and methionine metabolism. Alterations in metformin-induced longevity by mutation of worm methionine synthase (metr-1) and S-adenosylmethionine synthase (sams-1) imply metformin-induced methionine restriction in the host, consistent with action of this drug as a dietary restriction mimetic. Metformin increased or decreased worm lifespan, depending on E. coli strain, metformin sensitivity and glucose concentration.

Conclusions: In mammals, the intestinal microbiome influences host metabolism, including development of metabolic disease. Thus, metformin-induced alteration of microbial metabolism could contribute to therapeutic efficacy, and also to its side effects which include folate deficiency and gastrointestinal upset.

C. elegans is a small roundworm (nematode) usually found in the soil or in rotten fruits in temperate climates around the world. C. elegans feeds on diverse microorganisms like bacteria, whereas in the laboratory $C$. elegans is fed on $E$. coli and thus has only a single microbe. Active bacterial metabolism is a critical nutritional requirement for $C$. elegans; however, reduced bacterial metabolism retards development and extends its lifespan, probably through a process mimicking a dietary restriction-like state. Furthermore, worms with mutant $E$. coli have longer lifespans, suggesting that $E$. coli may play a more active role in $C$. elegans nutrition and metabolism than as a mere food source.

Metformin is widely used as an oral antihyperglycemic agent for T2DM and MetS. However, there is evidence that metformin also reduces the risk of cancer and, in animal models, delays aging. In this study, the authors examined the mechanism by which metformin extends lifespan in C. elegans. In a series of experiments the researchers showed that metformin increases the nematode lifespan, but only in worms that are fed on E. coli. Metformin induced a dose-dependent inhibition of E. coli proliferation and thus acted as a bacteriostatic on $E$. coli.

What characteristic of $E$. coli increases worm lifespan when altered by metformin? Since $C$. elegans was recently found to live longer on an $E$. coli mutant with reduced folate levels, and since metformin was found to decrease folate levels in patients, the authors assessed whether metformin increases worm lifespan by altering bacterial folate metabolism. Folates are B-group vitamins involved in the bacterial methionine cycle. Metformin, through its effect on folate metabolism and $E$. coli methionine metabolism, reduced methionine availability to the host $C$. elegans. Depletion of methionine is sufficient to mimic the dietary restricting lifespan effect. Interestingly, metformin did not extend lifespan on a high-glucose diet.

The authors suggest that their findings may have relevance to mammalian biology and human health. Bacteria in the human gut play a central role in nutrition and host biology, and affect the risk of obesity, MetS and T2DM. The finding that metformin influences the aging of C. elegans by altering microbial metabolism raises the possibility that this drug might similarly influence mammalian biology by affecting microbial metabolism or composition. 


\section{A form of the metabolic syndrome associated with mutations in DYRK1B}

Keramati AR, Fathzadeh M, Go GW, Singh R, Choi M, Faramarzi S, Mane S, Kasaei M, Sarajzadeh-Fard K, Hwa J, Kidd KK, Babaee Bigi MA, Malekzadeh R, Hosseinian A, Babaei M, Lifton RP, Mani A

Department of Internal Medicine, Yale Cardiovascular Research Center, Yale University School of Medicine, New Haven, CT, USA

N Engl J Med 2014;370:1909-1919

Introduction: Genetic analysis has successfully identified causative mutations for individual cardiovascular risk factors. Success has been more limited in mapping susceptibility genes for clusters of cardiovascular risk traits, such as the MetS.

Methods: Three large families with coinheritance of early-onset coronary artery disease, central obesity, hypertension, and diabetes were identified. Linkage analysis and whole-exome sequencing were used to identify the disease-causing gene.

Results: A founder mutation was identified in $D Y R K 1 B$, substituting cysteine for arginine at position 102 in the highly conserved kinase-like domain. The mutation precisely co-segregated with the clinical syndrome in all the affected family members and was absent in unaffected family members and unrelated controls. Functional characterization revealed that non-mutant protein encoded by DYRK1B inhibits the $\mathrm{SHH}$ (sonic hedgehog) and Wnt signaling pathways and consequently enhances adipogenesis. Furthermore, DYRK1B promoted the expression of the key gluconeogenic enzyme glucose-6-phosphatase. The MetS-associated allele R102C potentiated these effects by gain-of-function activities. A second mutation in DYRK1B, substituting proline for histidine 90, was found to co-segregate with a similar clinical syndrome in an ethnically distinct family.

Conclusions: These findings indicate a role for DYRK1B in adipogenesis and glucose homeostasis and associate its increased function with an inherited form of the MetS.

A substitution error in the gene DYRK1B segregated with abdominal obesity, hypertension, type 2 diabetes and early-onset coronary artery disease ( $<45$ years), running in three large families in Iran. In addition, a second substitution mutation in the same gene was found in members of a fourth ethnically distinct family, with morbid obesity, coronary artery disease and multiple metabolic phenotypes. The mutation was not found in unaffected family members or in ethnically-matched Iranian controls, nor from samples from several large DNA databases. The pattern of inheritance was consistent with a highly penetrant autosomal dominant trait.

$D Y R K 1 B$ encodes dual-specificity tyrosine-phosphorylation-regulated kinase $1 \mathrm{~B}$, which belongs to the Dyrk family of proteins, a group of evolutionarily conserved protein kinases that are involved in cell differentiation, survival and proliferation. Functional characterization revealed that DYRK1B protein normally enhances fat formation via inhibition of the SHH (sonic hedgehog) and Wnt signaling pathways. In addition, the DYRK1B gene appears to intensify the generation of glucose by increasing expression of the key enzyme glucose-6-phosphatase. Interestingly, increased expression and activity of glucose-6-phosphatase is associated with elevated fasting glucose levels in patients with T2DM. The R102C mutation discovered is a gain-of-function allele that boosts both effects. Such mutations in DYRK1B are likely to be rare, but genome-wide association studies have also linked common variants in DYRK1B to T2DM and traits associated with the metabolic syndrome in the general population. Approximately $3-4 \%$ of the population may carry these variants that impact MetS risk.

This study proposes that weight regulation is not governed by a uniform tally of 'calories in, calories out', but that there is a genetic or a biochemical component of obesity, and that weight is far from being entirely within an individual's control [7]. Furthermore, based on these findings, in the future a single drug may possibly target several metabolic risk factors. 


\section{Cortisol in hair and the metabolic syndrome}

Stalder T, Kirschbaum C, Alexander N, Bornstein SR, Gao W, Miller R, Stark S, Bosch JA, Fischer JE Department of Psychology, Technische Universität Dresden, Dresden, Germany

stalder@biopsych.tu-dresden.de

J Clin Endocrinol Metab 2013;98:2573-2580

Introduction: Exposure to supraphysiological levels of glucocorticoids contributes to the development of the metabolic syndrome (MetS). The importance of physiological variation in basal cortisol secretion is less clear.

Methods: Analysis of cortisol in hair (hairF) was used to examine associations between long-term cortisol levels and prevalence of MetS or individual cardiometabolic parameters in 1,258 subjects aged 16-64 years; $84.8 \%$ males. In addition, cardiometabolic associations with hair cortisone levels were studied. The first $3 \mathrm{~cm}$ of scalp-near hair were analyzed for glucocorticoid concentrations.

Results: Individuals in the third hairF quartile had 1.7-fold higher prevalence of MetS and those in the fourth quartile had a 2.4-fold higher prevalence of MetS compared with the first quartile. HairF showed positive associations with body mass index, waist-to-hip ratio, waist circumference and glycated hemoglobin. The exploratory analysis of hair cortisone also indicated relevant associations with cardiometabolic parameters.

Conclusion: Normal physiological differences in long-term cortisol secretion, as assessed in hair, show relevant relationships with MetS and individual cardiometabolic parameters.

Conditions of marked endogenous or iatrogenic hypercortisolism, such as Cushing's syndrome, closely resemble the symptom cluster of the MetS. This suggests that supraphysiological glucocorticoid levels may contribute to the development of cardiometabolic abnormalities. Data regarding the impact of physiological cortisol levels on MetS are controversial. While serum, salivary and urinary cortisol levels may reflect transient fluctuations, long-term glucocorticoid levels can be assessed in scalp hair. Lipophilic hormones incorporate into the growing hair, providing an index of integrated hormone levels over a period of several months.

The current study examined cortisol and cortisone levels in hair and their association with MetS. Overall, $24 \%$ of the participants met diagnostic criteria of the MetS. Those with MetS exhibited higher hair cortisol and cortisone levels and there was a dose response between higher hair cortisol levels and MetS prevalence. Specifically, hair glucocorticoid is associated with $\mathrm{HbA}_{1 \mathrm{c}}$ an index of long-term glucose levels, as well as with BMI, WHR and waist circumference.

Several important conclusions can be drawn. Firstly, long-term glucocorticoid levels appear to be associated with obesity and cardiometabolic measures. Secondly, the analysis of cortisol in hair is a highly promising method for the retrospective assessment of integrated cortisol secretion over extended periods of time and holds promise as a means of elucidating the role of steroid hormones in various diseases. Finally, the possibility of extending hair analysis to also capture long-term secretion of other steroid hormones, such as androgens and estrogens, provides an intriguing prospect for future research.

There is one obvious limitation of this method; exclusion criteria are baldness and obvious hair loss. However, other studies show that androgenic alopecia is positively associated with MetS and with higher risks of mortality from T2DM and heart disease [8]. 


\title{
Prolactin in obese children: a bridge between inflammation and metabolic- endocrine dysfunction
}

\author{
Chirico V, Cannavo S, Lacquaniti A, Salpietro V, Mandolfino M, Romeo PD, Cotta O, Munafo C, Giorgianni G, \\ Salpietro C, Arrigo T \\ Department of Pediatric Sciences, University of Messina, Messina, Italy \\ Clin Endocrinol (Oxf) 2013;79:537-544
}

Objective: Adipocytes, regulated by insulin, represent the major peripheral source of prolactin, which play a pivotal role in energy balance, acting on adipogenesis and lipolysis. This study aimed to investigate whether prolactin was associated with obesity-related inflammatory status and metabolic parameters. The diagnostic and prognostic role of prolactin for MetS was assessed. The effects of short-term lifestyle therapy on prolactin levels were evaluated.

Methods: Circulating prolactin levels were assessed in 94 obese patients and compared with 40 healthy children. An obesity intervention program was administered for 12 months.

Results: Prolactin levels were lower in obese patients than in controls $(\mathrm{p}<0.0001)$. Prolactin was inversely correlated with BMI, IL-6 and HOMA-IR, whereas a direct correlation was found with HDL values. Prolactin showed higher sensitivity and specificity than IL- 6 and TNF- $\alpha$ in identifying MetS in obese children and predicted MetS independently of other potential confounders. The lifestyle intervention increased prolactin levels and improved metabolic parameters.

Conclusions: Circulating prolactin represents a prognostic marker for obese children and a predictive factor for progression to MetS. Prolactin measurement may be useful as part of the endocrine work-up of obese children.

Pituitary-derived prolactin is a well-known regulator of the lactating mammary gland. The discovery that human adipose tissue also produces prolactin and expresses prolactin receptors indicated previously unappreciated actions in adipose tissue function [9]. Prolactin is secreted by macrophages in adipose tissue in response to inflammation and high glucose concentrations, acting as an adipokine. In a recent population-based study, an inverse association between prolactin levels and T2DM risk was demonstrated in both adult men and women [10]. In patients with polycystic ovary syndrome, prolactin levels were inversely associated with waist circumference, cholesterol and triglycerides, and positively associated with HDL. In the search for potential explanations, prolactin knockout and prolactin receptor deficiency were associated with reduced $\beta$-cell activity and glucose intolerance.

The current study assessed prolactin levels in obese children, its association with components of the MetS, and its changes during a lifestyle intervention. Prolactin levels were lower in obese than in normal weight children. Furthermore, prolactin levels were even lower in obese children with the MetS than in unaffected obese children. Prolactin was inversely correlated with BMI, IL-6 and HOMA-IR, and was positively correlated with HDL levels. Prolactin levels increased following lifestyle intervention in those who lost weight. Prolactin shows promise as a metabolic risk marker in obese children.

\section{Food for thought}

\section{Cardiorespiratory fitness and adiposity in metabolically healthy overweight and obese youth}

Senechal M, Wicklow B, Wittmeier K, Hay J, Maclntosh AC, Eskicioglu P, Venugopal N, McGavock JM

Manitoba Institute of Child Health, Winnipeg, MB, Canada

Pediatrics 2013;132:e85-e92

Objective: Controversy exists surrounding the contribution of fitness and adiposity as determinants of the metabolically healthy overweight (MHO) phenotype in youth. This study investigated the independent 
contribution of cardiorespiratory fitness and adiposity to the MHO phenotype among overweight and obese youth.

Methods: This cross-sectional study included 108 overweight and obese youth classified as MHO (no cardiometabolic risk factors) or non-MHO ( $\geq 1$ cardiometabolic risk factor), based on age- and genderspecific cut-points for fasting glucose, triglycerides, HDL-C, systolic and diastolic blood pressure, and hepatic steatosis.

Results: $25 \%$ of overweight and obese youth were classified as MHO. This phenotype was associated with lower BMI z-score (BMI z-score $1.8 \pm 0.3$ vs. $2.1 \pm 0.4, \mathrm{p}=0.02)$ and waist circumference $(99.7 \pm$ 13.2 vs. $106.1 \pm 13.7 \mathrm{~cm}, \mathrm{p}=0.04)$ compared with non-MHO youth. When matched for fitness level and stratified by BMI z-score (1.6 \pm 0.3 vs. $2.4 \pm 0.2)$, the prevalence of $\mathrm{MHO}$ was 4 -fold higher in the low BMI z-score group ( 27 vs. $7 \%$; $=0.03$ ). Multiple logistic regression analyses revealed that the best predictor of $\mathrm{MHO}$ was the absence of hepatic steatosis even after adjusting for waist circumference (OR 0.57, 95\% CI 0.40-0.80) or BMI z-score (OR 0.59, 95\% CI 0.43-0.80).

Conclusions: The MHO phenotype was present in $25 \%$ of overweight and obese youth and is strongly associated with lower levels of adiposity and the absence of hepatic steatosis, but not with cardiorespiratory fitness.

Obesity is a major public health problem associated with numerous metabolic disturbances, such as insulin resistance, T2DM, hypertension and dyslipidemia. However, these cardiometabolic abnormalities are not universal. A subset of obese people are apparently metabolically healthy (MHO). As many as $40 \%$ of the obese population have a favorable metabolic profile, characterized by normal insulin sensitivity, a low prevalence of hypertension, and favorable lipid and inflammation profiles. The converse condition, metabolically unhealthy normal weight (MUH-NW), has been coined to describe those individuals who are normal weight and yet have obesity-related metabolic abnormalities. About $4.6 \%$ of normal-weight men and $6.2 \%$ of normal-weight women have the MetS.

The current study assessed cardiorespiratory fitness and adiposity in $\mathrm{MHO}$ youth. The authors hypothesized that $\mathrm{MHO}$ youth have higher cardiorespiratory fitness. 108 subjects aged 13-18 years with BMI $>85$ centile were recruited. Cardiorespiratory fitness was quantified as $\mathrm{VO}_{2}$ peak relative to body weight and kilogram of fat-free mass. Physical activity was assessed by using segmented pedometry over a period of 7 days. The findings provide important insights into the determinants of cardiometabolic risk factor clustering among overweight and obese youth. Firstly, nearly $25 \%(n=27 / 108)$ of overweight and obese youth did not have a single cardiometabolic risk factor (i.e. were MHO); $43 \%$ had at least 1 risk factor, $33 \%$ had 2 risk factors, and $23 \%$ had MetS. Secondly, physical activity and cardiorespiratory fitness were not significantly different between $\mathrm{MHO}$ and other overweight and obese youth. The authors suggested that other modifiable lifestyle factors, including diet, sleep, and sedentary behavior, as well as non-modifiable risk factors, such as ethnicity, may contribute to the cardiometabolic risk factor clustering in overweight and obese youth.

\section{Lipid metabolism \\ Important for clinical practice}

\section{Treatment of blood cholesterol to reduce atherosclerotic cardiovascular disease risk in adults: synopsis of the 2013 American College of Cardiology/ American Heart Association cholesterol guideline}

Stone NJ, Robinson JG, Lichtenstein AH, Goff DC Jr, Lloyd-Jones DM, Smith SC Jr, Blum C, Schwartz JS; 2013 ACC/ AHA Cholesterol Guideline Panel

Ann Intern Med 2014;160:339-343

Background: In November 2013, the American College of Cardiology and American Heart Association (ACC/AHA) released a clinical practice guideline on the treatment of blood cholesterol to reduce cardiovascular risk in adults.

Methods: In 2008, the National Heart, Lung, and Blood Institute convened the Adult Treatment Panel (ATP) IV to update the 2001 ATP-III cholesterol guidelines using a rigorous process to systematically review randomized, controlled trials (RCTs) and meta-analyses of RCTs that examined cardiovascular 
outcomes. The panel commissioned independent systematic evidence reviews on LDL-C and non-highdensity lipoprotein cholesterol goals in secondary and primary prevention and the effect of lipid drugs on atherosclerotic cardiovascular disease events and adverse effects. In September 2013, the panel's draft recommendations were transitioned to the ACC/AHA.

Recommendations: This report summarizes key features of the guidelines in 8 areas: lifestyle, groups shown to benefit from statins, statin safety, decision-making, estimation of cardiovascular disease risk, intensity of statin therapy, treatment targets, and monitoring of statin therapy.

The 2013 ACC-AHA guidelines for the management of cholesterol substantially modified the previous recommendation that patients with established cardiovascular disease or diabetes and LDL-C $\geq 100 \mathrm{mg} / \mathrm{dl}$ were candidates for statin therapy. The use of statin therapy is now more widely recommended for primary prevention based of the combination of an individual's LDL-C level and 10-year risk of coronary heart disease as calculated by the Framingham risk score.

The new guidelines focus on treatment of blood cholesterol to reduce atherosclerotic cardiovascular disease (ASCVD) risk in adults. The first recommendation is adherence to healthy lifestyle behaviors. Statin therapy should be added as follows: (1) As secondary prevention for those with clinical ASCVD (acute coronary syndromes, myocardial infarction, stable angina, coronary or other arterial revascularization, stroke, transient ischemic attack, or peripheral arterial disease of atherosclerotic origin). (2) As primary prevention for: (a) adults with LDL-C $\geq 190 \mathrm{mg} / \mathrm{dl}(4.9 \mathrm{mmol} / \mathrm{l})$, (b) adults aged $40-75$ years with diabetes and LDL-C $\geq 70 \mathrm{mg} / \mathrm{dl}(1.8 \mathrm{mmol} / \mathrm{l})$, and (c) adults aged $40-75$ years without diabetes, with LDL-C 70-189 $\mathrm{mg} / \mathrm{dl}$ and 10-year ASCVD risk $\geq 7.5 \%$.

A clinician-patient discussion that considers potential ASCVD risk reduction, adverse effects, and patient preferences is needed to decide whether to initiate statin therapy, which may also be considered in adults aged 40-75 years without diabetes, with LDL-C 70-189 mg/dl and 10-year ASCVD risk 5 to $<7.5 \%$.

The 10-year estimated risk of cardiovascular disease is derived from a newly generated risk estimator that includes the following factors: age, sex, smoking status, total cholesterol level, HDL-C level, systolic blood pressure, the use of antihypertensive therapy and ethnicity. An online decision aid to help patients understand the benefits and harms of using statins to reduce their 10-year cardiovascular risk can be found at http://statindecisionaid.mayoclinic.org/index.php/statin/index.

Data used to estimate ASCVD risk reflect the risk profile of the general population, not of clinical trial cohorts or selective participation groups. Controversy emerged regarding the new 'pooled cohort equations'. Critics suggested that this risk calculator is poorly calibrated to recent cohorts and that the threshold for treatment (10-year ASCVD risk $\geq 7.5 \%$ ) is too low and should be $10 \%$ or higher.

\section{Application of new cholesterol guidelines to a population-based sample}

Pencina MJ, Navar-Boggan AM, D’Agostino RB Sr, Williams K, Neely B, Sniderman AD, Peterson ED

Duke Clinical Research Institute, Duke University, Durham, NC, USA

N Engl J Med 2014;370:1422-1431

Background: The 2013 ACC-AHA guidelines for the treatment of cholesterol expand the indications for statin therapy for the prevention of cardiovascular disease (CVD).

Methods: Using data from the National Health and Nutrition Examination Surveys of 2005-2010, the number of risk-factor profile of persons for whom statin therapy would be recommended (i.e. eligible persons) under the new ACC-AHA guidelines was compared with the guidelines of the ATP-III of the National Cholesterol Education Program, and extrapolated the results to a population of 115.4 million US adults aged between 40 and 75 years.

Results: Compared to the ATP-III guidelines, the new guidelines would increase the number of US adults receiving or eligible for statin therapy from 43.2 million $(37.5 \%)$ to 56.0 million (48.6\%). Most of this increase (10.4 of 12.8 million) would occur among adults without CVD. Among adults aged between 60 and 75 years without CVD who are not receiving statin therapy, the percentage who would be eligible for such therapy would increase from 30.4 to $87.4 \%$ among men and from 21.2 to $53.6 \%$ among women. This increase would be driven largely on the basis of their 10-year risk of a cardiovascular event. Those who would be newly eligible for statin therapy include more men than women, and adults with a higher blood pressure but a markedly lower level of low-density lipoprotein cholesterol. 
Compared to the previous guidelines, the new guidelines would recommend statin therapy for more adults with expected future cardiovascular events (higher sensitivity) but would also include many adults who would not have future events (lower specificity).

Conclusions: The new ACC-AHA guidelines would increase the number of adults eligible for statin therapy by 12.8 million. This increase is mostly among older adults without CVD.

The 2013 ACC-AHA guidelines were met with immediate intense criticism. Main concerns focused on flawed methods (validity of the risk calculator), ethics (conflicts of interest) and inferences (too many eligible for treatment) [11]. Indeed, this study calculated that the proportion of US adults eligible for statin therapy would rise to $48.5 \%$, from $37.5 \%$ under the previous ATP-III guidelines. This means an expected increase of 12.8 million adults eligible for statin therapy under the new guidance. Most of the increase (10.4 million) would occur in adults without cardiovascular disease (primary prevention) and in those in the upper end of the age range (60-75 years). Notably, the median LDL-C among newly eligible adults would be only $105.2 \mathrm{mg} / \mathrm{dl}(2.7 \mathrm{mmol} / \mathrm{l})$. Finally, when the data were extrapolated to similar age groups around the globe, a rough estimate of 920 million people would be classified as eligible for statin therapy [11]!

\section{Clinical trials, new treatments}

\section{Mipomersen, an apolipoprotein B synthesis inhibitor, reduces atherogenic lipoproteins in patients with severe hypercholesterolemia at high cardiovascular risk: a randomized, double-blind, placebo-controlled trial}

Thomas GS, Cromwell WC, Ali S, Chin W, Flaim JD, Davidson M

Long Beach Memorial Medical Center, Long Beach, and University of California, Irvine, CA, USA

gthomas1@memorialcare.org

J Am Coll Cardiol 2013;62:2178-2184

Background: Many patients on lipid-lowering therapies remain unable to achieve target low-density lipoprotein cholesterol (LDL-C) levels. Mipomersen, an antisense oligonucleotide inhibitor of apolipoprotein B, reduces LDL-C and atherogenic lipoproteins. This study aimed to examine the efficacy and safety of mipomersen to reduce atherogenic lipids and lipoproteins in patients with hypercholesterolemia.

Methods: This randomized, double-blind, multicenter study enrolled 158 patients with baseline LDL-C levels $\geq 100 \mathrm{mg} / \mathrm{dl}$ with, or at high risk for, coronary heart disease who were receiving maximally tolerated lipid-lowering therapy. Patients received weekly subcutaneous mipomersen $200 \mathrm{mg}(\mathrm{n}=105)$ or placebo $(\mathrm{n}=52)$ for 26 weeks, with a 24 -week follow-up period. Randomization was stratified by T2DM.

Results: 60 mipomersen and 44 placebo patients completed treatment. Mean baseline LDL-C levels were 122.7 and $122.6 \mathrm{mg} / \mathrm{dl}$ in the placebo and mipomersen patients, respectively. Mipomersen reduced LDL-C by $-36.9 \%$ compared with placebo at $-4.5 \%(\mathrm{p}<0.001)$. Target LDL-C $<100 \mathrm{mg} / \mathrm{dl}$ was attained in $76 \%$ of mipomersen and $38 \%$ of placebo patients. Mipomersen also significantly reduced apolipoprotein B $(-38 \%)$ and lipoprotein $(\mathrm{a})(-24 \%)(\mathrm{p}<0.001)$. Common adverse events included injection site reactions ( $78 \%$ with mipomersen, $31 \%$ with placebo) and flu-like symptoms $(34 \%$ with mipomersen, $21 \%$ with placebo). Elevations in transaminases and liver fat also occurred in some patients, and these levels returned toward baseline after treatment cessation.

Conclusions: Mipomersen reduced LDL-C, apolipoprotein B, and lipoprotein(a) in patients with hypercholesterolemia with, or at risk for, coronary heart disease not controlled by existing therapies.

Antisense oligonucleotides bind to and modulate the function of targeted RNA through diverse post-binding mechanisms, which in general either interfere with RNA function or promote RNA degradation [12]. They represent a promising drug platform with the potential to selectively target any RNA in the transcriptome. In January 2013, the US FDA approved mipomersen for homozygous familial hypercholesterolemia. Mipomersen is a second-generation antisense oligonucleotide that 
inhibits the synthesis of apolipoprotein $B-100$ (apoB-100) by binding to apoB mRNA, to form a substrate for ribonuclease $\mathrm{H}$, a ubiquitously expressed nuclease that preferentially hydrolyzes the RNA strand of a RNA-DNA duplex. Apolipoprotein B (apoB) is constitutively expressed in the liver and is an essential component of LDL-C, very-low-density lipoprotein (VLDL), intermediate-density lipoprotein, and lipoprotein(a) (Lp). Conventional lipid-lowering therapies often result in insufficient LDL-C reductions, even at maximally tolerated doses. This phase 3 study assessed the safety and efficacy of mipomersen in patients with hypercholesterolemia and with a high-risk coronary heart disease who had already received a maximally tolerated lipid-lowering regimen. Efficacy of mipomersen was seen as early as 5 weeks, resulting in greater reductions from baseline in mean LDL-C $(-36.9 \%)$ than placebo-treated patients $(-4.5 \%)$. These effects were similar in patients with T2DM and even greater in women and older patients. However, due to a high frequency of adverse effects, including injection site reactions, flu-like symptoms, liver function test abnormalities, and hepatic steatosis, $43 \%$ of mipomersen-treated subjects dropped out. Interestingly, among patients with homozygous familial hypercholesterolemia, the dropout rate was only $18 \%$, suggesting a lower sense of treatment urgency in patients with lower baseline LDL and without known genetic diseases.

\section{Follow-up on a Yearbook 2012 paper}

\section{A 52-week placebo-controlled trial of evolocumab in hyperlipidemia}

Blom DJ, Hala T, Bolognese M, Lillestol MJ, Toth PD, Burgess L, Ceska R, Roth E, Koren MJ, Ballantyne CM, Monsalvo ML, Tsirtsonis K, Kim JB, Scott R, Wasserman SM, Stein EA; DESCARTES Investigators

Division of Lipidology, Department of Medicine, University of Cape Town, Cape Town, and TREAD Research, Cardiology Unit, Department of Internal Medicine, Tygerberg Hospital and Stellenbosch University, Parow, South Africa; Center for Clinical and Basic Research, Pardubice, and Center of Preventive Cardiology,

Third Department of Internal Medicine, Charles University, Prague, Czech Republic; Bethesda Health Research Center, Bethesda, MD, Lillestol Research, Fargo, ND, Midwest Institute for Clinical Research, Indianapolis, IN, Sterling Research Group and Metabolic and Atherosclerosis Research Center, Cincinnati, OH, Jacksonville Center for Clinical Research, Jacksonville, FL, Baylor College of Medicine and the Houston Methodist DeBakey Heart and Vascular Center, Houston, TX, and Amgen, Thousand Oaks, CA, USA, and Amgen, Uxbridge, UK N Engl J Med 2014;370:1809-1819

Background: Evolocumab, a monoclonal antibody that inhibits proprotein convertase subtilisin/kexin type 9 (PCSK9), reduced LDL-C levels in phase 2 studies. A phase 3 trial to evaluate the safety and efficacy of 52 weeks of treatment with evolocumab was conducted.

Methods: According to the ATP-III classification, during the 4- to 12 week run-in period, patients with hyperlipidemia were started on background lipid-lowering therapy with diet alone or diet + atorvastatin at a dose of $10 \mathrm{mg}$ daily, atorvastatin at a dose of $80 \mathrm{mg}$ daily, or atorvastatin at a dose of $80 \mathrm{mg}$ daily + ezetimibe at a dose of $10 \mathrm{mg}$ daily. Patients with an LDL level of $\geq 75 \mathrm{mg} / \mathrm{dl}(1.9 \mathrm{mmol} / \mathrm{l})$ at the end of the run-in period were then randomly assigned in a 2:1 ratio to receive either evolocumab (420 $\mathrm{mg}$ ) or placebo every 4 weeks. The primary endpoint was the percent change from baseline to week 52 in LDL-C, as measured by ultracentrifugation.

Results: Among the 901 patients included in the primary analysis, the mean $( \pm \mathrm{SE})$ reduction in LDL-C from baseline in the evolocumab group, taking into account the change in the placebo group, was $57.0 \pm 2.1 \%(\mathrm{p}<0.001)$. The mean reduction was $55.7 \pm 4.2 \%$ among patients on background therapy with diet alone, $61.6 \pm 2.6 \%$ among those on $10 \mathrm{mg}$ of atorvastatin, $56.8 \pm 5.3 \%$ among those on 80 $\mathrm{mg}$ of atorvastatin, and $48.5 \pm 5.2 \%$ among those on a combination of $80 \mathrm{mg}$ of atorvastatin and 10 $\mathrm{mg}$ of ezetimibe ( $\mathrm{p}<0.001$ for all comparisons). Evolocumab treatment also reduced levels of apolipoprotein $\mathrm{B}$, non-HDL-C, lipoprotein(a), and triglycerides. The most common adverse events were nasopharyngitis, upper respiratory tract infection, influenza, and back pain.

Conclusions: At 52 weeks, evolocumab added to diet alone, to low-dose atorvastatin, or to high-dose atorvastatin with or without ezetimibe significantly reduced LDL-C levels in patients with a range of cardiovascular risks. 
Proprotein convertase subtilisin kexin type 9 (PCSK9) is synthesized primarily in the liver and enters the circulatory system where it binds to and accelerates the degradation of hepatic LDL receptors. This process reduces the capacity of the liver to remove LDL-C from the circulation. Patients with PCSK9 gain-of-function mutations have very high LDL-C levels, whereas individuals with PCSK9 lossof-function mutations have unusually low LDL-C levels and low incidence of coronary heart disease. Previous short-term (8- to 12-week), placebo-controlled, phase 2 trials showed that evolocumab, a monoclonal antibody-specific inhibitor of PCSK9, significantly reduced LDL-C levels.

The current study tested evolocumab against placebo in patients with hyperlipidemia. They received the study drug for 52 weeks after a run-in period of 4-12 weeks of background lipid-lowering therapy. A total of 901 participants received at least one dose of a study drug, and $800(88.4 \%)$ completed 52 weeks of treatment.

Treatment with evolocumab 420 mg once monthly for 1 year resulted in a relative reduction in LDL-C levels of $57 \%$. The recommended target of LDL-C $\leq 70 \mathrm{mg} / \mathrm{dl}$ was achieved in more than $80 \%$ of patients. The overall incidence of adverse events was similar in the evolocumab and the placebo groups. PCSK9 inhibition with the human monoclonal antibody evolocumab appears to be an attractive and effective strategy to reduce LDL-C and improve lipid profiles. A longer-term cardiovascular outcome trial is ongoing.

\section{Food for thought}

\section{A statin a day keeps the doctor away: comparative proverb assessment modelling study}

Briggs A, Mizdrak A, Scarborough P

BHF Health Promotion Research Group, Nuffield Department of Population Health, University of Oxford, Oxford, UK BMJ 2013;347:f7267

Objective: To model the effect on UK vascular mortality of all adults over 50 years of age being prescribed either a statin or an apple a day.

Methods: In a comparative proverb assessment modelling study, either a statin a day for people not already taking a statin or an apple a day for everyone, assuming $70 \%$ compliance and no change in calorie consumption. Main outcome measure was mortality due to vascular disease.

Results: The estimated annual reduction in deaths from vascular disease with a statin a day, assuming $70 \%$ compliance and a relative reduction in vascular mortality of $12 \%$ (95\% CI 9-16\%) per 1.0 $\mathrm{mmol} / \mathrm{l}$ reduction in low density lipoprotein cholesterol, is 9,400 . The equivalent reduction with an apple a day, assuming an apple weighs $100 \mathrm{~g}$, is 8,500.

Conclusions: Both nutritional and pharmaceutical approaches to the prevention of vascular disease may have the potential to significantly reduce UK mortality. With similar reductions in mortality, a 150 -yearold health promotion message is able to match modern medicine and is likely to have fewer side effects.

BMJ's Christmas Edition is well known for its humor and parody. In their introduction the authors state that: 'An apple a day keeps the doctor away, a public health message delivered by parents and teachers since the 19th century, is an example of how concise, clear, and accurate Victorian health promotion truly stood the test of time. In contrast, other Victorian practices - such as the use of leeches in primary care - have fallen away'.

Using mathematical modelling, the authors estimated the effect on vascular mortality of prescribing everybody in the UK over 50 years old either an apple a day or a statin a day. Side effects modeled for statin use included an incidence of myopathy of $0.5 / 1,000$ patients over 5 years, of rhabdomyolysis of $0.1 / 1,000$ over 5 years, and of T2DM of $0.1 \%$ per year, assuming $70 \%$ compliance. Side effects modeled for increased apple consumption included the distress caused by a bruised apple and the theoretical risk of finding half a worm inside. Costs were modeled too; simvastatin $40 \mathrm{mg}$ at GBP 1.24 /month was compared to the 4.7 pence/apple, assuming that the apples are on the smaller side. Prescribing either an apple a day or a statin a day is likely to have a similar effect on population vascular mortality. Choosing apples rather than statins may avoid more than 
a 1,000 excess cases of myopathy and more than 12,000 excess diabetes diagnoses. The authors concluded that a 150-year-old proverb is able to match modern medicine and is likely to have fewer side effects.

\section{References}

1. Dabelea D, Mayer-Davis EJ, Lamichhane AP, D'Agostino RB Jr, Liese AD, Vehik KS, et al: Association of intrauterine exposure to maternal diabetes and obesity with type 2 diabetes in youth: the SEARCH Case-Control Study. Diabetes Care 2008;31:1422-1426.

2. Dabelea D, Hanson RL, Bennett PH, Roumain J, Knowler WC, Pettitt DJ: Increasing prevalence of type 2 diabetes in American-Indian children. Diabetologia 1998;41:904-910.

3. Dabelea D, Mayer-Davis EJ, Saydah S, Imperatore G, Linder B, Divers J, et al: Prevalence of type 1 and type 2 diabetes among children and adolescents from 2001 to 2009. JAMA 2014;311:1778-1786.

4. Zeitler P, Hirst K, Pyle L, Linder B, Copeland K, Arslanian S, et al: A clinical trial to maintain glycemic control in youth with type 2 diabetes. N Engl J Med 2012;366:2247-2256.

5. Hanghoj S, Boisen KA: Self-reported barriers to medication adherence among chronically ill adolescents: a systematic review. J Adolesc Health 2014;54:121-138.

6. Tamborlane WV, Klingensmith G: Crisis in care: limited treatment options for type 2 diabetes in adolescents and youth. Diabetes Care 2013;36:1777-1778.

7. Jou C: The biology and genetics of obesity - a century of inquiries. N Engl J Med 2014;370:1874-1877.

8. Su LH, Chen LS, Lin SC, Chen HH: Association of androgenetic alopecia with mortality from diabetes mellitus and heart disease. JAMA Dermatol 2013;149:601-606.

9. Brandebourg T, Hugo E, Ben-Jonathan N: Adipocyte prolactin: regulation of release and putative functions. Diabetes Obes Metab 2007;9:464-476.

10. Balbach L, Wallaschofski H, Volzke H, Nauck M, Dorr M, Haring R: Serum prolactin concentrations as risk factor of metabolic syndrome or type 2 diabetes? BMC Endocr Disord 2013;13:12.

11. Ioannidis JP: More than a billion people taking statins? Potential implications of the new cardiovascular guidelines. JAMA 2014;311:463-464.

12. Bennett CF, Swayze EE: RNA targeting therapeutics: molecular mechanisms of antisense oligonucleotides as a therapeutic platform. Annu Rev Pharmacol Toxicol 2010;50:259-293. 